\title{
Lupus Mastitis as Differential Diagnosis of Breast Mass
}

\author{
João Antonio Pessôa Corrêa, Maria Célia Resende Djahjah
}

Department of Radiology, Clementino Fraga Filho University Hospital of the Federal University of Rio de Janeiro, RJ, 21941-590, Brazil.

\section{Corresponding Author:}

Dr. João Antonio Pessôa Corrêa

Email: joaoapcorrea@gmail.com

This is an Open Access article distributed under the terms of the Creative Commons Attribution License (creativecommons.org/ licenses/by/3.0).

Received

Accepted

Published

April 29, 2020

July 18,2020

August 10, 2020

\begin{abstract}
Background: Lupus mastitis is a rare presentation of lupus panniculitis, seen in up to 2 to $3 \%$ of the patients and rarely being the initial presentation of systemic lupus erythematosus. It affects the deep subcutaneous adipose tissue of the breast. Case Report: A previously healthy 36-year-old white woman presented with a history of a palpable mass in the right breast that had developed over one year. Physical examination revealed a palpable mass with skin retraction, local erythema and an ulcer in healing in the right breast. Imaging evaluation with mammography and ultrasound classified the findings as suspicious, indicating biopsy. Biopsy revealed findings suggestive of lupus mastitis. Patient was treated with anti-malarial drugs, with clinical improvement. Conclusion: The findings on physical examination and imaging studies of lupus mastitis can be misleading. Depending on the presentation on the onset, it can mimic breast infection or even breast carcinoma. Magnetic Resonance Imaging is useful in evaluating the extent of disease and may have an important role in monitoring treatment response. Histopathology has an important role in suggesting diagnosis, leading to the correct treatment and avoiding erroneous surgical procedures, which can work as triggers for new episodes of mastitis.
\end{abstract}

Keywords: Adult, Breast, Breast Neoplasms, Female, Lupus Erythematosus, Panniculitis.

\section{Introduction}

Lupus panniculitis (LP) or lupus erythematosus profundus is an uncommon inflammatory presentation in patients with systemic lupus erythematosus (SLE) or discoid lupus, first described by Kaposi in 1883. Lupus mastitis (LM) is a rare presentation of LP involving the deep subcutaneous adipose tissue of the breast, rarely being the initial presentation of SLE [1-3]. LM often presents both clinically and mammographically as a mass. Suspicious calcifications can also be detected on mammography. Acutely, LM is usually painful and the skin can appear erythematous or violaceous. The course of the disease is chronic, and some lesions can ulcerate or become atrophic, but others will resolve [3]. The aim of this case report is to describe a new case of LM, since there are few case reports in literature, discussing the role of imaging and pathology in the differential diagnosis of this breast mass.

\section{Case Report}

A previously healthy 36-year-old white woman presented with a history of a palpable mass in the right breast that had developed over one year. There was a report of an ulcer on the site of the mass one month prior, treated with antibiotics, with partial resolution. On physical examination, a palpable mass occupying the upper and lower inner quadrants of the right breast was present, with skin retraction, local erythema and an ulcer in healing in the union of the inner quadrants [Fig.1]. There was no nipple discharge and the axillae were free, with no palpable lymph node. There were no relevant findings on the left breast. 
Mammography demonstrated skin thickening and retraction of the inner quadrants of the right breast, architectural distortion in the central region and a small round and circumscribed nodule on the upper outer quadrant [Fig.2]. Ultrasound showed an ill-defined hypoechoic area in the central region, skin thickening, and a round and circumscribed nodule in the outer upper quadrant. Both examinations were categorized as Breast Imaging Reporting and Data System (BI-RADS) 4, and ultrasound-guided biopsy was suggested. Dynamic contrast-enhanced magnetic

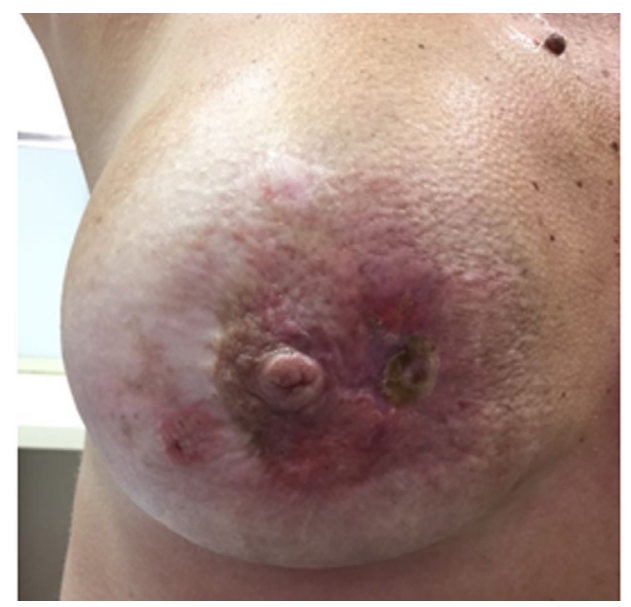

Fig.1: Right breast presenting with skin retraction, local erythema and an ulcer in healing in the union of the inner quadrants.

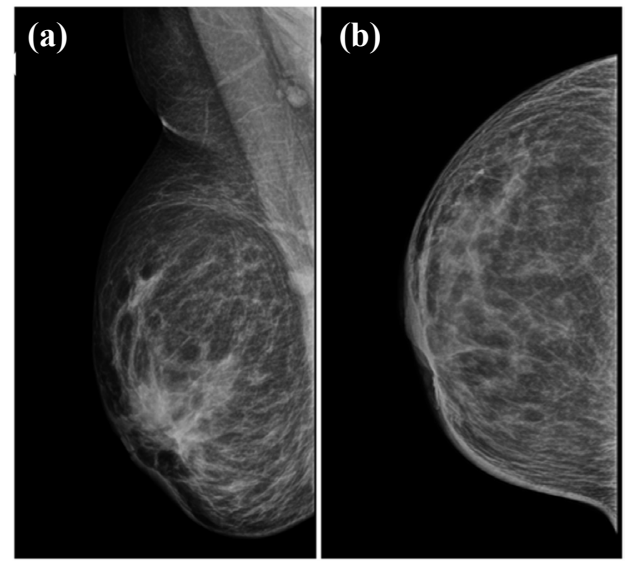

Fig.2: Mammography (a) medio-lateral oblique and (b) cranio-caudal views showing skin thickening and retraction of the inner quadrants of the right breast and architectural distortion in the central region. resonance imaging (MRI) was performed after the histopathologic results, showing skin thickening, retraction and contrast enhancement, architectural distortion in the retro-areolar region with heterogeneous, moderate and ascending contrast enhancement (type I kinetic curve) in association with local edema [Fig.3a,b]. Restricted diffusion was not observed in the area of interest. Abnormal lymph nodes were present, with homogeneous thickening of the cortical region, and were regarded as an inflammatory reaction. All the findings were categorized as BI-RADS 2.

Core biopsy of the nodule suggested intra-mammary lymph node. Histopathological examination of the skin and adjacent breast tissue suggested the diagnosis of lupus mastitis. Blood work-up was performed, with positive ANA (1:160) showing a speckled pattern. Anti-dsDNA, anti-Smith, anti-Ro/SSA, anti-La/SSB were negative and complement levels were normal. The patient was then treated with anti-malarial drugs, with clinical improvement. The final diagnosis was lupus mastitis without systemic disease.

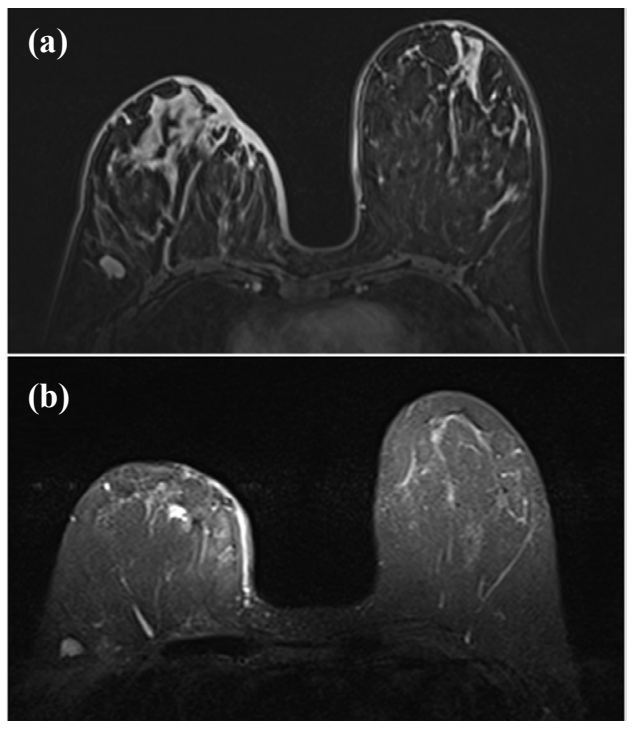

Fig.3:(a) Axial contrast-enhanced T1-weighted fat suppressed MRI, first phase, with skin thickening, retraction and enhancement; lymph node with homogeneous thickening of the cortical region and non-mass like enhancement with mild background parenchymal enhancement; (b) Axial STIR sequence MRI showing edema of the inner quadrants of the right breast. 


\section{Discussion}

Panniculitis is an acute or chronic inflammatory condition affecting the subcutaneous tissue and can occur in association with systemic diseases, including SLE. When associated with SLE, it has been denominated LP, and breast involvement is termed LM. LM is an uncommon finding in SLE, seen in up to 2 to $3 \%$ of the patients [4] with few case reports of LM as the first manifestation of SLE $[3,5]$.

The most commonly described radiological findings at mammography were large and dystrophic calcifications usually associated with fat necrosis seen in advanced LM; masses, often ill-defined, or asymmetries, focal or diffuse. On ultrasound, ill-defined hypoechoic masses, architectural distortions and changes in echotexture, usually hyperechoic resulting from infiltration, were the most common findings [3,5]. Skin thickening and axillary lymph node enlargement did not occur as often [3]. In comparison, our case didn't have calcification at mammography, but the other findings at mammography and ultrasound, both common and uncommon, were found.

There were few studies describing LM MRI findings in the literature. The main features described are skin thickening, fat stranding and heterogeneous contrast enhancement, with variable reports of enhancement curves $[3,6]$. Our findings are similar in comparison, with type I kinetic curve, usually indicative of benignity. The imaging aspects of LM that we have so far in literature are not specific to the disease, hindering LM diagnosis when using imaging findings alone, especially when it appears without associated systemic disease. The differential diagnosis of inflammatory carcinoma must be ruled out with histopathological study since there is no absolute imaging finding that differentiates it from LM [3]. MRI is most useful in assessing the extent of disease, including skin involvement, and monitoring treatment response
$[3,6,7]$. Mosier et al. suggest that MRI may be useful in evaluating treatment response since decreasing thickness of the peripheral enhancement appears to be correlated with clinical improvement [7].

Histopathological criteria for the diagnosis of lupus mastitis include four major and minor criteria. The major criteria include hyaline fat necrosis, lymphocytic infiltration with lymphoid nodules surrounding the necrosis, periseptal or lobular panniculitis and microcalcifications. The minor criteria include discoid changes in overlying skin, lymphocytic vasculitis, mucin deposition, and hyalinization of sub-epidermal papillary lesions. $[3,6]$. The presence of all the criteria is not mandatory for diagnosis. $[3,6]$.

After diagnosis, the use of steroids and anti-malarial drugs are the ideal therapy $[2,3]$. Surgical procedures must be avoided at most, since surgical trauma may be an inciting event in LP [1]. The findings on physical examination and imaging studies can be misleading. Depending on the presentation on the onset, it can mimic breast infection or even breast carcinoma. With few cases reported in the literature, it is hard to suggest LM and rule out malignancy with imaging findings alone. MRI is useful in evaluating the extent of disease and may have an important role in monitoring treatment response. Histopathology has an important role in suggesting the diagnosis, leading to the correct treatment and avoiding erroneous surgical procedures, which can work as a trigger for new episodes of mastitis.

\section{Conclusion}

Physical examination and image findings can be inconclusive, being hard to suggest LM or rule out malignancy with this alone. Histopathology is important in suggesting the diagnosis, avoiding unnecessary intervention causing recurrence of LM. MRI is effective when evaluating the extent of disease and may help in monitoring treatment response. 
Contributors: JAPC, MCRD: Drafting the work and revising it critically for important intellectual content and the conception of the work. JAPC will act as a study guarantor. Both authors approved the final version of this manuscript and are responsible for all aspects of this study.

Funding: None; Competing interests: None stated.

\section{References}

1. Rosa M, Mohammadi A. Lupus mastitis: a review. Ann Diagn Pathol. 2013;17:230-233.

2. Summers T, Lehman M, Barner R, Royer M. Lupus mastitis. Adv Anat Pathol. 2009;16:56-61.

3. Voizard B, Lalonde L, Sanchez L, Chesney JR, David J, Labelle M, et al. Lupus mastitis as a first manifestation of systemic disease: About two cases with a review of the literature. Eur J Radiol. 2017;92:124-131.

4. Kinonen C, Gattuso P, Reddy V. Lupus mastitis. Am J Surg Pathol. 2010;34:901-906.

5. Warne RR, Taylor D, Segal A, Irish A. Lupus mastitis: a mimicker of breast carcinoma. BMJ Case Rep. 2011; 2011: bcr1120115066.

6. Thapa A, Parakh A, Arora J, Goel R. Lupus mastitis of the male breast. BJR case reports. 2016;2:20150290.

7. Mosier A, Boldt B, Keylock J, Smith D, Graham J. Serial MR findings and comprehensive review of bilateral lupus mastitis with an additional case report. J Radiol Case Rep. 2013;7:48-58. 